\title{
Chronic active EBV infection in refractory enteritis with longitudinal ulcers with a cobblestone appearance: an autopsied case report
}

\author{
Yosuke Aihara ${ }^{1}$, Kei Moriya ${ }^{2^{*}}$ (D, Naotaka Shimozato ${ }^{1,2}$, Shinsaku Nagamatsu', Shinya Kobayashi ${ }^{3}$, \\ Masakazu Uejima ${ }^{1}$, Hideki Matsuo ${ }^{1}$, Eiwa Ishida ${ }^{4}$, Hideo Yagi ${ }^{3}$, Toshiya Nakatani ${ }^{1}$, Hitoshi Yoshiji ${ }^{2}$ \\ and Eiryo Kikuchi ${ }^{1}$
}

\begin{abstract}
Background: Chronic active Epstein-Barr virus infection (CAEBV) is defined as Epstein-Barr virus (EBV)-positive T/NK cell-related neoplasia, and its major clinical symptom is systemic inflammation presenting as infectious mononucleocytosis, whereas enteritis and diarrhea are minor clinical symptoms. The complex mixture of tumorigenic processes of EBV-positive cells and physical symptoms of systemic inflammatory disease constitutes the varied phenotypes of CAEBV. Herein, we describe a case of CAEBV that was initially diagnosed as Crohn's disease (CD) based on ileal ulcers and clinical symptoms of enteritis.
\end{abstract}

Case presentation: A 19-year-old woman complained of abdominal pain and fever. Blood examination showed normal blood cell counts without atypical lymphocyte but detected modest inflammation, hypoalbuminemia, slight liver dysfunction, and evidence of past EBV infection. The esophagogastroduodenoscopic findings were normal. However, colonoscopy revealed a few small ulcers in the terminal ileum. The jejunum and ileum also exhibited various forms of ulcers, exhibiting a cobblestone appearance, on capsule endoscopy. Based on these clinical findings, she was strongly suspected with CD. In the course of treatment by steroid and biologics for refractory enteritis, skin ulcers appeared about 50 months after her initial hospital visit. Immunohistology of her skin biopsy revealed proliferation of EBVencoded small RNA (EBER)-positive atypical lymphocytes. We retrospectively assessed her previous ileal ulcer biopsy before treatment and found many EBER-positive lymphocytes. Blood EBV DNA was also positive. Therefore, she was diagnosed with extranodal NK/T-cell lymphoma with CAEBV-related enteritis rather than CD. She was treated with cyclosporine and prednisolone combination therapy for CAEBV-related systemic inflammation and chemotherapy for malignant lymphoma. Unfortunately, her disease continued to progress, leading to multiple organ failure and death at the age of 23 years.

Conclusion: Clinicians need to remember the possibility of CAEBV as a differential diagnosis of refractory enteritis. Enteritis with intestinal ulcer is a rare symptom of CAEBV, and it is impossible to acquire a definitive diagnosis by ulcer

\footnotetext{
*Correspondence: moriyak@naramed-u.ac.jp

2 Department of Gastroenterology and Hepatology, Nara Medical

University, 840 Shijo-cho, Kashihara, Nara 634-8522, Japan

Full list of author information is available at the end of the article
} original author(s) and the source, provide a link to the Creative Commons licence, and indicate if changes were made. The images or other third party material in this article are included in the article's Creative Commons licence, unless indicated otherwise in a credit line to the material. If material is not included in the article's Creative Commons licence and your intended use is not permitted by statutory regulation or exceeds the permitted use, you will need to obtain permission directly from the copyright holder. To view a copy of this licence, visit http://creativecommons.org/licenses/by/4.0/. The Creative Commons Public Domain Dedication waiver (http://creativeco mmons.org/publicdomain/zero/1.0/) applies to the data made available in this article, unless otherwise stated in a credit line to the data. 
morphology only. In cases where the possibility of CAEBV remains, tissue EBVR expression should be checked by in situ hybridization and blood EBV DNA.

Keywords: Crohn's disease, Cobblestone appearance, Inflammatory bowel disease, Refractory enteritis, Chronic active EBV infection, Lymphoproliferative disorders, Malignant lymphoma, Biologics, Capsule endoscopy, Case report

\section{Background}

Enteritis is an inflammatory disease of the small intestine caused by some factors, such as bacterial or viral infections, ischemia, vasculitis, chemical or radiological tissue damage, and immune disorders including inflammatory bowel disease (IBD), with phenotype variation [1]. In fact, few cases of chronic active Epstein-Barr virus (CAEBV) reveal enteritis [2-4]. Previously, CAEBV was thought to be a rare child disease limitedly occurring in the East Asia. However, CAEBV has been recently found to occur worldwide with no relation to patient's age [5]. Although CAEBV is a phenotype of EBV-lymphoproliferative disorders, its major clinical symptom is systemic inflammation presenting as infectious mononucleosis (IM) and enteritis as well as diarrhea are minor clinical symptoms. The complex mixture of tumorigenic processes associated with EBV-positive cells and the physical symptoms of inflammatory systemic disease constitute the varied phenotypes of CAEBV. The median period between the first visit and the definite diagnosis of CAEBV was 20 months. This extended duration was mainly due to the need to first exclude other diseases or cases where patients were diagnosed with unknown fever [6]. Although CAEBV generally presents with IM-like symptoms, we recently experienced a case of a young woman with CAEBV that appeared with refractory enteritis and was indistinguishable from Crohn's disease (CD). This report of an autopsied CAEBV case will be of educational help, given the difficulty in acquiring a definite diagnosis.

\section{Case presentation}

A 19-year-old woman was referred to our hospital for intermittent abdominal pain and continuous fever which had persisted for a month. She had no obvious medical or familial history. Physical examination revealed no morbid lymph node swelling or skin abnormalities. Blood examination showed normal blood cell counts and no presence of atypical lymphocytes. Biochemical or immunoserum analysis revealed modest inflammation, hypoalbuminemia, slight liver dysfunction, and evidence of past EBV infection (Additional file 1: Table S1). Although esophagogastroduodenoscopy revealed normal findings, colonoscopy revealed a few small ulcers in the terminal ileum (Additional file 3: Fig. 1). Enhanced computed tomography showed obvious wall thickness of the small intestine, whereas no significant finding was detected by balloon intestinal endoscopy in the distal ileum. However, various types of ulcers surrounded by completely normal mucosa were found on the jejunum and proximal ileum by capsule endoscopy (CE) (Fig. 1). Additionally, multiple aphthous and some linear ulcers were observed on the jejunum, whereas circular ulcers and longitudinal ulcers with a cobblestone appearance were detected on the ileum. The possibility of intestinal tuberculosis and infectious gastroenteritis were serologically and culturally excluded. Although there were no histological findings of noncaseating granuloma on her digestive organs, she was strongly suspected with $C D$ based on her age, clinical symptoms, and the morphology of the characteristic ulcers (Fig. 2). Consequently, the patient was treated with an elemental diet, mesalamine, and prednisolone for induction therapy. Although this treatment seemed to be partially effective, her symptoms recurred according to the tapering of prednisolone, and she was subsequently switched to adalimumab (ADA). Her clinical symptoms tended to be gradually modest, and the maintenance ADA therapy was effective for some time. The second CE revealed a definite improvement of the intestinal ulcers (Fig. 3a, b); however, 21 months after the initiation of treatment, her intermittent fever and repeated abdominal pain recurred, and re-induction therapy with prednisolone was restarted (Fig. 2). She remained stable for 9 months, before complaining of slight abdominal pain. We set a dose escalation of ADA with the addition of azathioprine; this seemed to be effective, and almost all of the ulcers disappeared, except for a few small erosions on the intestine (Fig. 3c, d). She next relapsed approximately 10 months later, and ADA was switched to ustekinumab (USK). However, this treatment was not sufficient, and prednisolone was added to USK for clinical improvement. Unfortunately, disease management was difficult regardless of these treatments, and skin ulcers appeared about 50 months after her initial hospital visit (Fig. 4a). Immunohistology of her skin biopsy revealed proliferation of EBV-encoded small RNA (EBER)-positive atypical lymphocytes (Fig. 4b, c). Retrospective assessments of the previous ileal ulcer biopsy before treatment demonstrated many EBER-positive lymphocytes, indicating that she had been in a continuously active EBV infection state (Fig. 4d, e). The blood level of EBV-DNA was also clearly positive $\left(1.5 \times 10^{4}\right.$ copy/WBC $\left.\times 10^{6}\right)$. Consequently, she was diagnosed with extranodal NK/T-cell lymphoma (nasal type) 

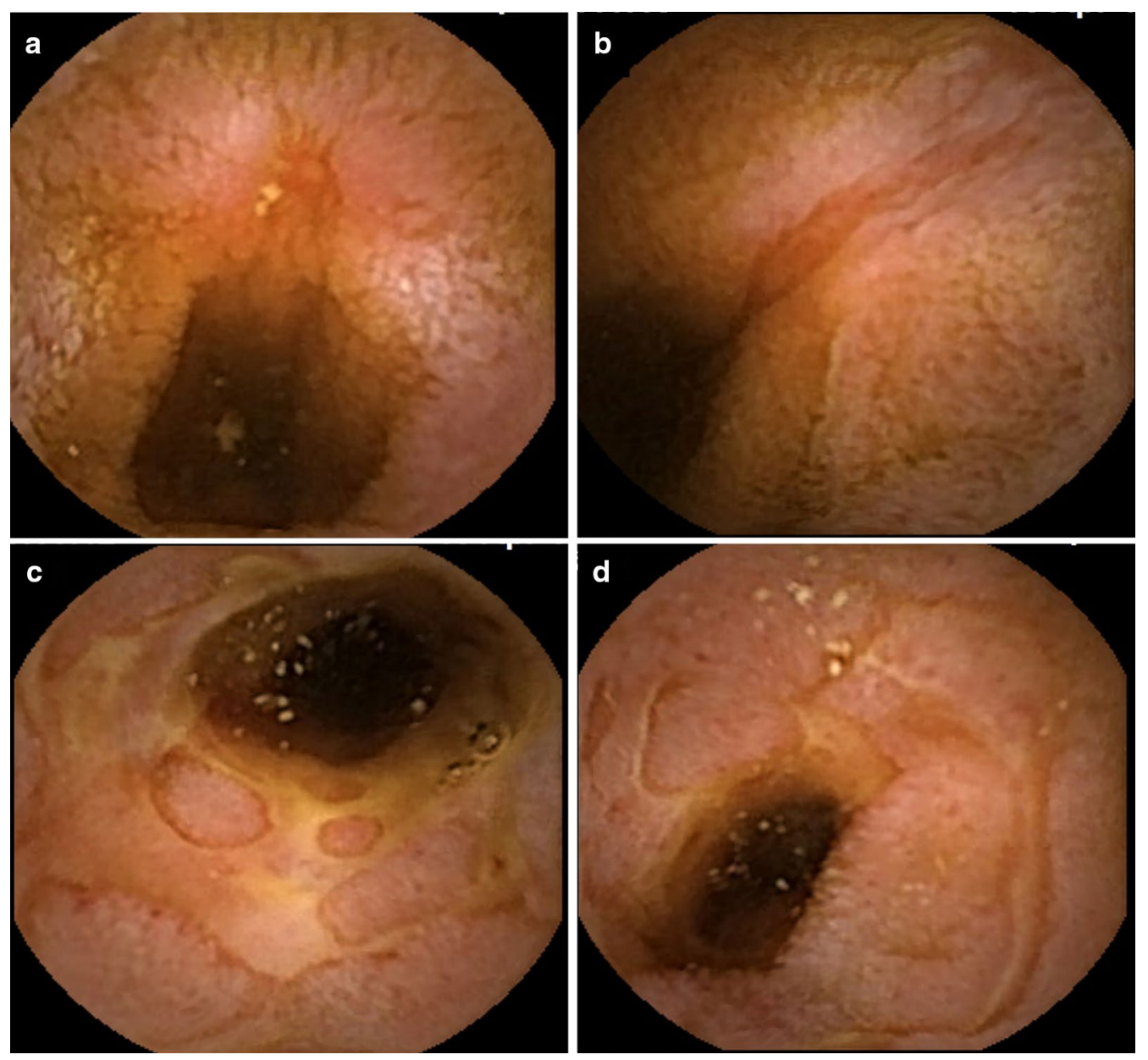

Fig. 1 Various forms of ulcers on digestive organs detected by capsule endoscopy prior to treatment. The surrounding mucosa was free from inflammation. a Multiple aphthous ulcers on the jejunum. b Liner ulcers on the jejunum. $\mathbf{c}$ Longitudinal ulcers with a cobblestone appearance on the ileum. $\mathbf{d}$ Circular ulcers on ileum

related to CAEBV. Advanced-stage malignant lymphoma invading the skin was seen. Then, she was treated with a combination of cyclosporine A and prednisolone for her systemic inflammation related to CAEBV and followed by a systemic chemotherapy for malignant lymphoma. After completing the CHOP chemotherapy (cyclophosphamide, doxorubicin, vincristine, and prednisolone), she gradually showed intermediate liver injury as well as cholestasis, based on which she was diagnosed with secondary hemophagocytic lymphohistiocytosis (HLH) caused by CAEBV. Though etoposide and methylprednisolone combination therapy transiently improved her liver injury and jaundice, CHOP insufficiently controlled the progression of CAEBV. Hence, the combination therapy of etoposide, cytosine arabinoside, $\mathrm{L}$-asparaginase, methylprednisolone, and prednisolone (ESCAP) was preferred over the $\mathrm{CHOP}$ therapy. However, her liver injury and jaundice related to HLH rapidly worsened during the recovery period after the first ESCAP therapy. Unfortunately, her disease progression could not be regulated at all and she finally fell into the stage of multiple organ failure; she died at the age of 23 years, 55 months after her first hospital visit. An autopsy was performed with the consent from her parents. Macroscopic findings showed no evidence of ulcer or stenotic changes on her digestive organs, including the small intestine (Fig. 5a). Microscopic findings showed no evidence of noncaseating granuloma on digestive organs and a remarkable proliferation of lymphocytes in the intra-mucosal/submucosal space of the small intestine (Fig. 5b, c). 


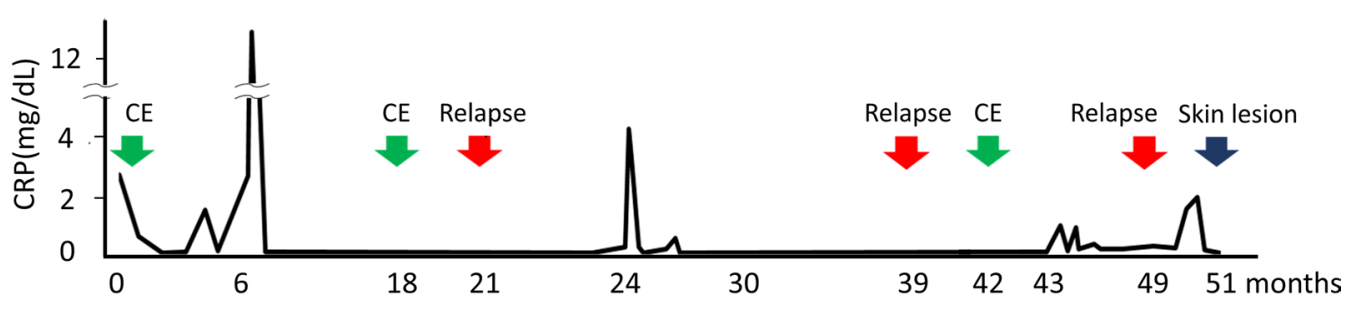

Start of treatment

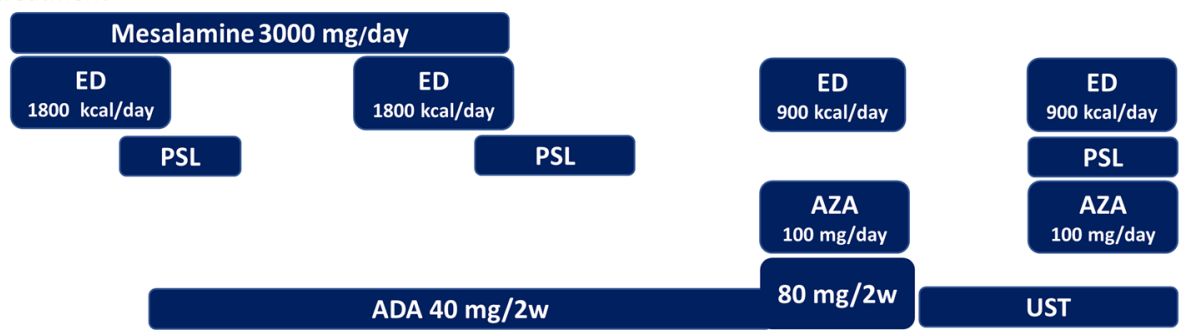

ED: Elemental diet, PSL: Prednisolone, ADA: Adalimumab, AZA: Azathioprine, UST: Ustekinumab

Fig. 2 Entire clinical course of the present case

\section{Discussion and conclusion}

Chachu and Osterman concluded that "Thoughtful consideration and evaluation of these other potential etiologies through patient history and physical examination, laboratory tests, endoscopic evaluation with targeted biopsies of lesions, and cross-sectional imaging is required to evaluate any patient presenting with symptoms consistent with IBD" [7]. However, it is difficult to completely distinguish CAEBV enteritis from IBD by endoscopic findings only, and it is important to comprehend the clinical features of each disease. To appropriately diagnose CAEBV, it is important to remember the possibility of CAEBV as a differential diagnosis of systemic inflammation [8]. When CAEBV is suspected, the EBV-DNA level in peripheral blood should be checked, even in immunologically denied cases of acute EBV infection. Identification of EBV-infected $\mathrm{T}$ and NK cells should be performed for definite diagnosis of CAEBV. Moreover, in situ hybridization (ISH) of EBER by pathological examination and EBV-DNA analysis by flow cytometry should be performed. More details are described in the latest diagnostic criteria of CAEBV [5, 9].

To our knowledge, the first report of a case with gastrointestinal tract complications of CAEBV had serious colorectal bleeding [10]. Later, in the 2000s, there were several reports of CAEBV with gastrointestinal tract complications, which had initially been misdiagnosed as IBD [2-4]. Approximately 6\% CAEBV-related deaths originated from intestinal bleeding or perforation [6]. As summarized in the Additional file 2: Table S2, the ulcer morphology of "cobblestone appearance" characteristic of $C D$ [11] was first found in our current case, but this was not observed in all 27 previously reported CAEBVrelated enteritis cases $[2-4,10,12,13]$. According to the endoscopic features of these 28 cases, the diseased lesion was located in the colon (13 cases), small intestine (five cases), concomitant colon and small intestine (five cases), concomitant colon and ileocecal junction (one case), concomitant colon and stomach (one case), ileocecal junction (one case), and unknown location (two cases). Ulcer morphologies have generally been reported as small, shallow, irregular-shaped, and scattered, with the exception of eight cases of huge and profound ulcers. There was also a previous case in which the entire intestinal mucosa displayed lymphangiectasia [3]. In our case, many small aphthous ulcers were found in the whole intestine, and a cobblestone appearance was observed, especially in the ileum, whereas no ulcer was found in the colon. These aphthous ulcers remained after intensive treatment with steroids and biologics. Based on these findings, CAEBV-related enteritis might present various ulcer morphologies depending on the reaction of each therapeutic treatment and/or time course. To detect intestinal lesions, we adopted CE, which has been increasingly used worldwide since its establishment in 2000 [14]. With the advent of CE, various diseases have been clearly visualized, resulting in a paradigm shift in the diagnosis and treatment of small bowel disease. According to a nationwide study in Japan, the frequent findings of $\mathrm{CE}$ in patients with $\mathrm{CD}$ include cobblestone appearance (occurrence rate: 33\%), longitudinal ulcer (78\%), irregular ulcer (84\%), liner erosion (90\%), irregular erosion (89\%), circumferential alignment of diminutive 

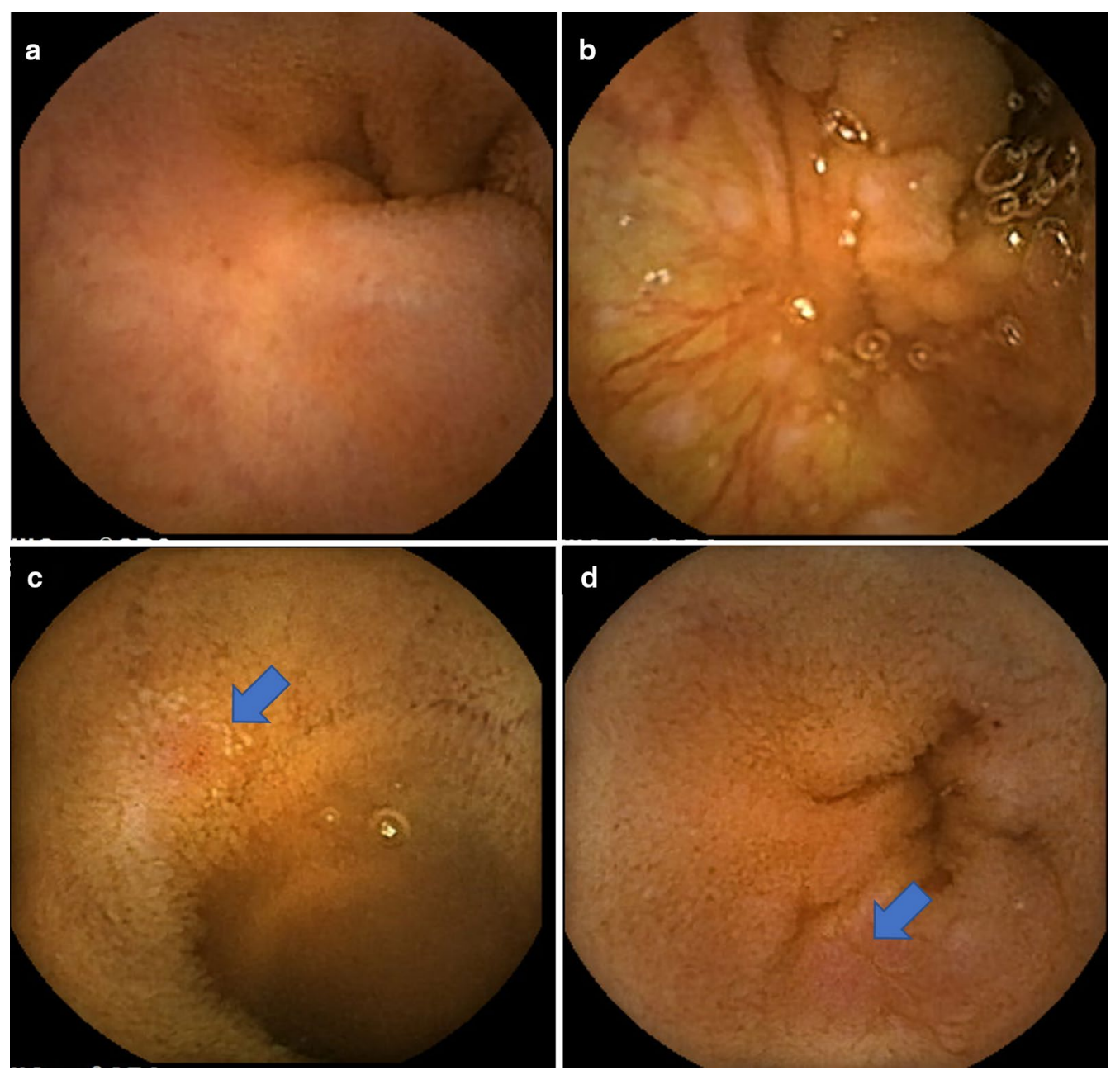

Fig. 3 Findings of the second capsule endoscopic examination. $\mathbf{a}, \mathbf{b}$ Ulcer scars on the jejunum and ileum (18 months after the first treatment). $\mathbf{c}$, $\mathbf{d}$ Small recurrent erosions on the jejunum and ileum (42 months after the first treatment)

lesions (75\%), and longitudinal alignment of diminutive lesions (56\%) [15]. In this previous study, Esaki et al. observed that the endoscopic diagnosis varied in endoscopist's clinical knowledge and proficiency; hence, this issue should be overcome. In this case, CD was most probable because ulcers with a cobblestone appearance were detected in the ileum. However, other characteristic findings, such as the bamboo joint-like appearance of the gastric mucosa in esophagogastroduodenoscopy, focally enhanced gastritis, and granuloma on histological evaluation were not actually confirmed.

Thus, a final diagnosis should be comprehensively made based on physical symptoms, medical histories, clinical findings, and culture, imaging, and pathological tests. Even if the characteristic endoscopic findings of specific enteritis are detected, differential diagnosis based only on these findings will be difficult because the characteristic findings of endoscopy do not always correspond to the specific disease. Some enteric diseases might show similar endoscopic appearances, whereas the same disease might demonstrate various phenotypes depending on the time course and severity.

Unfortunately, no successful treatment for CAEBV has yet been established, and further research is needed to improve the outcome in the future $[16,17]$. Therefore, it is crucial for clinicians to definitely diagnose CAEBV as quickly as possible.

In conclusion, we diagnosed a rare case of CAEBV, with refractory enteritis as the main clinical symptom and without manifestation of a typical IM feature. Diagnosing CAEBV by ulcer morphology is difficult, even with $C E$ and routine histopathological examination only; thus, clinicians should consider CAEBV as a differential diagnosis of refractory enteritis in younger patients and eagerly check blood EBV-DNA and EBER expression levels by ISH. 

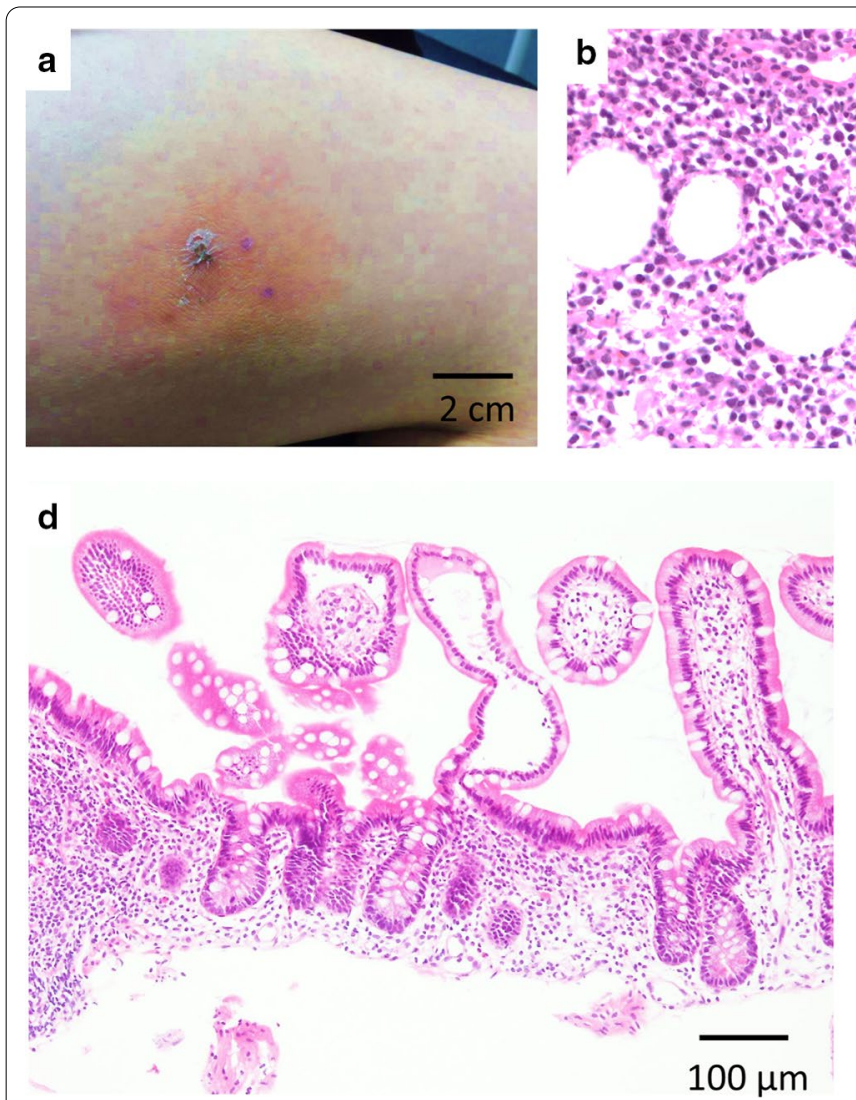
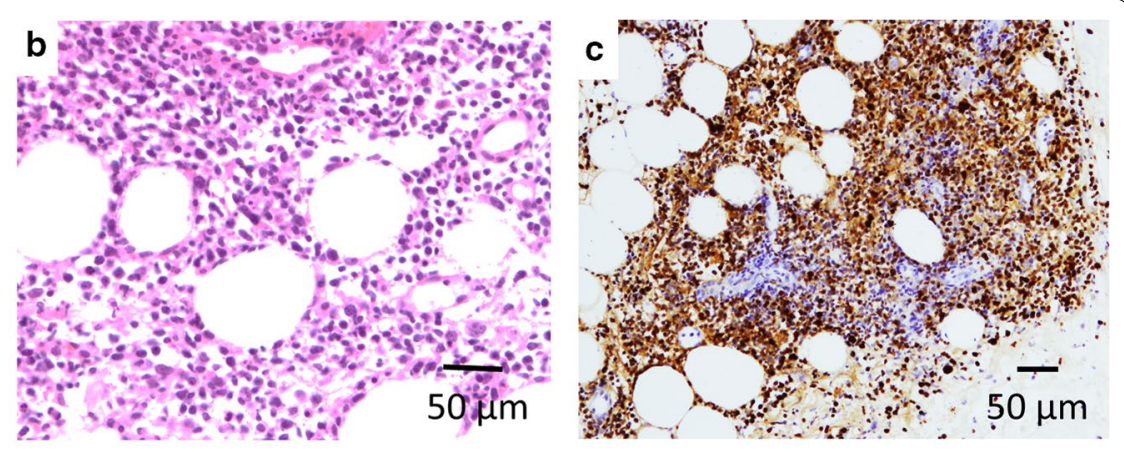

e

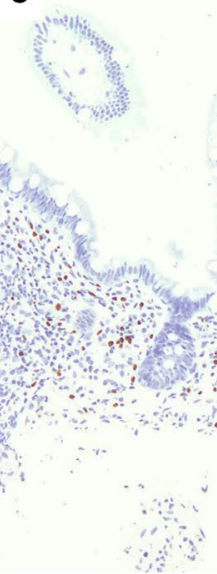

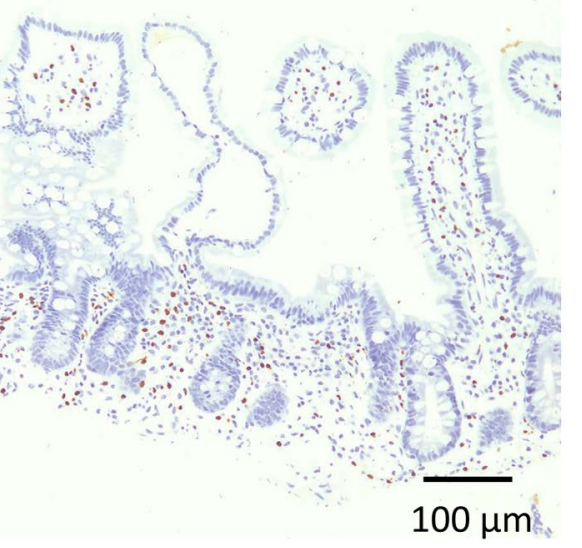

Fig. 4 Histological findings of extranodal NK/T-cell lymphoma (nasal type) associated with continuously activated EBV infection. a Macroscopic finding of skin ulcer on the patient's thigh, which occurred more than 4 years after the initiation of medical treatment. $\mathbf{b}$ Microscopic finding of H\&E staining of the skin ulcer. c Microscopic findings revealed high proliferation of EBER-positive atypical lymphocytes in the diseased skin. $\mathbf{d}$ Microscopic finding of H\&E staining in the intestinal ulcer. e Microscopic immunohistological findings of ileal ulcerative lesions revealed moderate infiltration of many EBER-positive lymphocytes, which demonstrated continuously active EBV infection
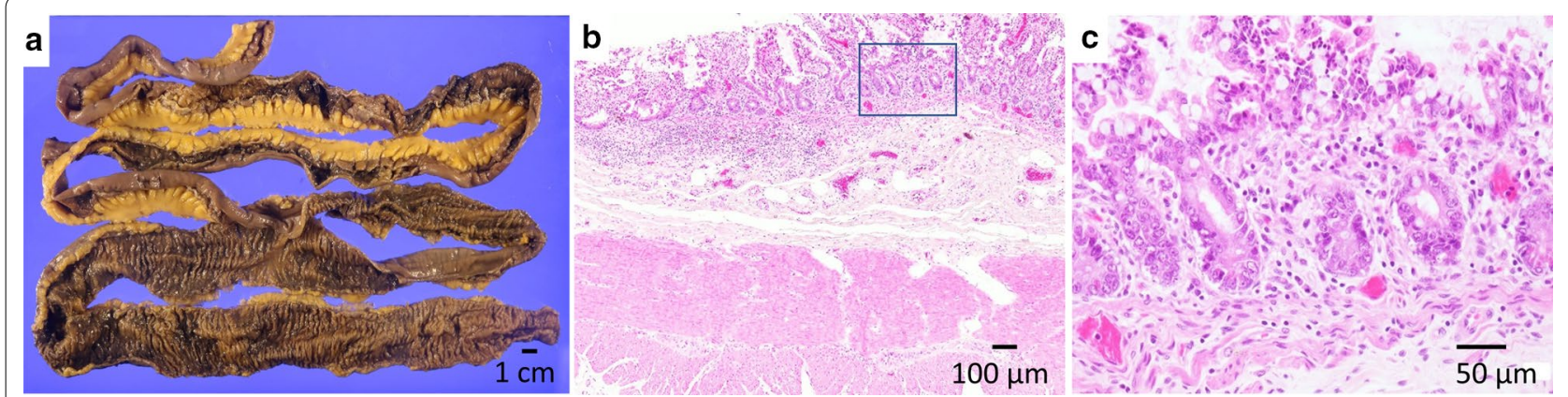

Fig. 5 Histological findings of the autopsied small intestine. a Macroscopic observation revealed neither ulcerative changes nor stenotic changes in the small intestine. $\mathbf{b}$ Microscopic finding of H\&E staining of the intestinal tissue. c Microscopic observation revealed mild lymphocyte proliferation with no atrophy of the intestinal villi (large magnification of H\&E staining)

\section{Patient perspective}

The patient's mother kindly told the authors that the patient had decided to fight her disease, though she had well realized the features and general prognosis of CAEBV. 


\section{Supplementary Information}

The online version contains supplementary material available at https://doi. org/10.1186/s12876-020-01589-1.

Additional file 1: Supplementary Table S1. Results of her blood test on the first admission.

Additional file 2: Supplementary Table S2. Clinical features of the reported CAEBV cases.

Additional file 3: Figure S1. Endoscopic findings of each digestive organ before treatment. (a) Esophagus, (b) stomach, (c) duodenum, (d) terminal ileum, (e) colon, and (f) rectum. Arrow indicates an ulcerative lesion on the terminal ileum.

\section{Abbreviations}

CAEBV: Chronic active Epstein-Barr virus infection; EBV: Epstein-Barr virus; CD: Crohn's disease; IBD: Inflammatory bowel disease; IM: Infectious mononucleosis; CE: Capsule endoscopy; ADA: Adalimumab; USK: Ustekinumab; EBER: EBV-encoded small mRNA; ISH: In situ hybridization; HLH: Hemophagocytic lymphohistiocytosis.

\section{Acknowledgements}

The authors deeply appreciate the mercy of the patient and her parents for accepting the autopsy and publication of this report on her illness.

\section{Authors' contributions}

Writing: YA and KM. Editing: KM. Figure preparation: NS, SN, SK, MU, HM, El, and $H$ Yagi. Supervision: TN, H Yoshiji, and EK. All authors have read and approved the final version of this manuscript.

\section{Funding}

There is nothing to declare.

\section{Availability of data and materials}

Data on this case not reported in the manuscript are available from the corresponding author upon reasonable request.

\section{Ethics approval and consent to participate}

Ethics committee approval is not required for a single presentation retrospectively. In accordance with the "Patient Rights Regulation" (Date: 01.08.1998; Issue: 23420), which was prepared in accordance with international standards, verbal and wet signed written consent was obtained from the patient under the title of "Consent to publish"to publish patient information and support public health.

\section{Consent for publication}

The parents of the patient provided written consent for reporting her case in an international published medical journal, including clinical details and images.

\section{Competing interests}

The authors confirm that there are no conflicts of interest to declare.

\section{Author details}

${ }^{1}$ Department of Gastroenterology and Hepatology, Nara Prefecture General Medical Center, Nara, Japan. ${ }^{2}$ Department of Gastroenterology and Hepatology, Nara Medical University, 840 Shijo-cho, Kashihara, Nara 634-8522, Japan. ${ }^{3}$ Department of Hematology, Nara Prefecture General Medical Center, Nara, Japan. ${ }^{4}$ Department of Pathology, Nara Prefecture General Medical Center, Nara, Japan.
Received: 18 September 2020 Accepted: 21 December 2020

Published online: 06 January 2021

\section{References}

1. Gecse KB, Vermeire S. Differential diagnosis of inflammatory bowel disease: imitations and complications. Lancet Gastroenterol Hepatol. 2018:3:644-53.

2. Roth DE, Jones A, Smith L, Lai R, Preiksaitis J, Robinson J. Severe chronic active Epstein-Barr virus infection mimicking steroid-dependent inflammatory bowel disease. Pediatr Infect Dis J. 2005;24:261-4.

3. Tseng YJ, Ding WQ, Zhong L, Chen J, Luo ZG. Chronic active Epstein-Barr virus (CAEBV) enteritis. Int J Infect Dis. 2019;82:15-7.

4. Zhang Y, Jiang Z, Liu R, Chen H, Wang M, Cao Q. Chronic active EpsteinBarr Virus associated enteritis may develop into a precancerous disease. Inflamm Bowel Dis. 2017;23:19-21.

5. Arai A. Advances in the study of chronic active Epstein-Barr virus infection: clinical features under the 2016 WHO classification and mechanisms of development. Front Pediatr. 2019:7:14

6. Arai A, Imadome KI, Watanabe Y, et al. Clinical features of adult-onset chronic active Epstein-Barr virus infection: a retrospective analysis. Int J Hematol. 2011;93:602-9.

7. Chachu KA, Osterman MT. How to diagnose and treat IBD mimics in the refractory IBD patient who does not have IBD. Inflamm Bowel Dis. 2016:22:1262-74.

8. Okano M, Kawa K, Kimura H, et al. Proposed guidelines for diagnosing chronic active Epstein-Barr virus infection. Am J Hematol. 2005:80:64-9.

9. Swerdlow SH, Campo E, Pileri SA, et al. The 2016 revision of the World Health Organization classification of lymphoid neoplasms. Blood. 2016;127:2375-90

10. Okano M, Thiele GM, Davis JR, Nauseef WM, Mitros F, Purtilo DT. Adenovirus type-2 in a patient with lethal hemorrhagic colonic ulcers and chronic active Epstein-Barr virus infection. Ann Intern Med. 1988;108:693-9.

11. Nikolaus S, Schreiber S. Diagnostics of inflammatory bowel disease Gastroenterol. 2007;133:1670-89.

12. Liu R, Wang M, Zhang L, et al. The clinicopathologic features of chronic active Epstein-Barr virus infective enteritis. Mod Pathol. 2019;32:387-95.

13. Xu W, Jiang $X$, Chen J, et al. Chronic active Epstein-Barr virus infection involving gastrointestinal tract mimicking inflammatory bowel disease. BMC Gastroenterol. 2020;20:257.

14. Iddan G, Meron G, Glukhovsky A, Swain P. Wireless capsule endoscopy. Nature. 2000;405:417.

15. Esaki M, Matsumoto T, Ohmiya N, et al. Capsule endoscopy findings for the diagnosis of Crohn's disease: a nationwide case-control study. J Gastroenterol. 2019:54:249-60.

16. Kimura H, Ito Y, Kawabe S, et al. EBV-associated T/NK-cell lymphoproliferative diseases in non-immunocompromised hosts: prospective analysis of 108 cases. Blood. 2012;119:673-86.

17. Sawada A, Inoue M, Kawa K. How we treat chronic active Epstein-Barr virus infection. Int J Hematol. 2017;105:406-18.

\section{Publisher's Note}

Springer Nature remains neutral with regard to jurisdictional claims in published maps and institutional affiliations. 